\title{
ACCIÓN PEDAGÓGICO-SOCIAL CON MENORES QUE TIENEN DIFICULTADES EN LA SOCIALIZACIÓN
}

\author{
José Vicente Merino Fernández \\ Universidad Complutense de Madrid
}

\begin{abstract}
RESUMEN. Este trabajo contribuye a avanzar en una pedagogía de la inclusión desde una perspectiva teórica y otra práctica. En la teórica analiza y muestra como los menores, que por las causas que fuere tienen dificultades en su proceso de socialización, comportan o adquieren necesidades educativas especiales, y necesitan consecuentemente una ayuda educativa específica. La aportación práctica consiste en ofrecer al investigador profesional:

1. Elementos teóricos y metodológicos para el diseño de la acción pedagógicosocial como una unidad de acción interdisciplinaria y personalizada.

2. Tres instrumentos prácticos para el desarrollo y evaluación de la acción pedagógico-social:

a) Principios inspiradores y reguladores de la acción pedag6gico-social.

b) Esquema guía para facilitar la elaboración de adaptaciones curriculares individuales.

c) Constructo teórico-práctico en el que se especifican los pasos para el estudio de caso como modelo de intervención individual.
\end{abstract}

SUMMARY. A The main goal of this study is to explore and ponder the contribution of the social-pedagogical action to a education and reeducation of the minor whith socialization problems.

For this porpuse the author analyses and develops the minor with socializations problems like people who bear or acquire a special or exceptional educative needs. Also, the author offers three practice implements:

1. Some principles of the social-pedagogical actions.

2. A scheme or guide to elaborate a individual curriculum system (adaptaciones curriculares individuales) or the program's individualization.

3. A theoretical-practical construct to show the steps of a the "individual case" like a individual intervention pattern or model.

\section{Planteamiento}

A nadie se le oculta que las personas con limitaciones individuales o en desamparo $y$ dificultad social se enfrentan en desigualdad de condiciones al reto de la socialización. La concepción integral del ser humano y su derecho a la vida y a la 
educación exige una ayuda individual y social específica para quienes, por las razones que fuere, están más indefensos o encuentran mayores dificultades que la media en su socialización. La acción preventiva se manifiesta en esta tarea como prioritaria sobre la curativa, punitiva y correctiva, y por consiguiente, la educación como acción preventiva por excelencia adquiere una importancia preponderante.

La educación es un derecho de toda persona humana. La mayoría de los estados modernos reconocen y regulan este derecho. Así queda explicitado en el Pacto Internacional de Derechos Económicos, Sociales y Culturales, aprobado por la O.N.U. el 16-XII-1966, firmado por España el 28-IX-1976, y ratificado el 13-IV-1977 (B.O.E. 30-IV-1977). El artículo 27 de la Constitución española actual no sólo recoge este derecho en la frase "todos tienen el derecho a la educación" $(27,1)$, sino que considera que "la educación tendrá por objeto el pleno desarrollo de la personalidad humana" $(27,2)$.

El Fondo internacional de las Naciones Unidas de ayuda a la infancia, U.N.I.C.E.F., en su principio VII, explícita este derecho, e insiste en el desarrollo individual y social del niño como el eje sobre el que ha de disponerse y ejecutarse la educación:

El niño tiene derecho a recibir educación, que será gratuita y obligatoria por lo menos en las etapas elementales. Se le dará una educación que favorezca su cultura general y le permita, en condiciones de igualdad de oportunidades, desarrollar sus aptitudes y su juicio individual, su sentido de responsabilidad moral y social y llegar a ser un miembro útil de la sociedad.

El interés superior del niño debe ser el principio rector de quienes tienen la responsabilidad de su educación y de su orientación; dicha responsabilidad incumbe en primer lugar a sus padres.

La Ley Orgánica 8/1985, de 3 de julio, Reguladora del Derecho a la Educación (LODE) concreta este derecho cuando manifiesta de manera explícita en el artículo $6^{\circ}$ que los alumnos que por diferentes razones requieran una ayuda específica para poder educarse, el Estado habrá de proporcionárselo:

Se reconoce a los alumnos los siguientes derechos básicos:

a) Derecho a recibir una formación que asegure el pleno desarrollo de su personalidad.

- (..).

g) Derecho a recibir las ayudas precisas para compensar posibles carencias de tipo familiar, económico y sociocultural.

h) Derecho a protección social en los casos de infortunio familiar o accidente $(6,1)$.

La educación como proceso de perfeccionamiento de la totalidad integral de la persona humana en sus dimensiones individuales y sociales incluye al proceso de socialización, considerado éste como "proceso interaccional de aprendizaje social" (J. Merino, 1984 , 145). La socialización, como mostré en el trabajo citado, no es un proceso de acomodación a las situaciones standard de la sociedad en la que se vive, y por lo tanto, un proceso pasivo, sino un proceso activo a través del cual el individuo ha de lograr su identidad personal, su identidad social y su inserción social. En el logro del primer obje- 
tivo (identidad personal) predominan los procesos intelectuales y psíquico individuales, y son fundamentalmente introspectivos; en el segundo (identidad social) sobresalen todos aquellos procesos comunicativos y de adquisición de habilidades, competencias y roles sociales, y en el tercero (incorporación social) se busca no solo la incorporación socio-laboral sino también su realización y proyección personal a través de diferentes contribuciones personales, culturales y laborales que como miembro activo y responsable aporta a la sociedad. Los dos primeros son de maduración personal y social, mientras que el tercero es fundamentalmente proyectivo.

Este modelo integral de socialización superador de las explicaciones sociologistas e individualistas, constituye y potencia un proceso de aprendizaje social en el que se busca y pretende la armonía entre las posibilidades y limitaciones del individuo y los condicionamientos y requerimientos del medio social. Intervienen, en comunicación e interinfluencia activa, tanto el propio individuo como el medio social y cultural. Para que esta acción interactiva sea educación es necesario conseguir el protagonismo activo del individuo como agente principal del propio proceso, al tiempo que lograr que este mismo proceso perfeccione al individuo.

El objetivo de la acción pedagógica es garantizar dicho protagonismo, adecuando, coordinando, gestionando, y en algunos casos dirigiendo, todas aquellas situaciones, factores y procesos necesarios para que la armonía entre individuo y contexto no se rompa. El desarrollo de una educación social adecuada a cada edad y situación personal y social será el mejor medio para posibilitar al individuo una socialización educativa.

La acción pedagógica con menores que tienen dificultades en la socialización, como tal acción pedagógica, ha de generar y desarrollar, e incluso ser en sí misma, educación social. La previsión y regulación de las dificultades, así como el control de las mismas cuando ya surgieron, o la compensación de las limitaciones innatas o adquiridas, que dificultan u obstaculizan la socialización, serán tareas de la acción pedagógica con el fin de responder con rigor científico y sensibilidad humana a este derecho de los menores.

El principio es bonito. La realidad social es más cruda tanto a nivel de actitudes como de condicionamientos obstaculizantes. Conseguir que no quede en puro deseo o en una bella utopía requiere un esfuerzo de toda la sociedad y no sólo del sistema formal educativo o de las familias afectadas. Como tarea de toda la sociedad, implica acciones políticas, económicas, comunitarias y familiares coordinadas. Las diferencias, por exceso o por defecto, y la especificidad de numerosos individuos y grupos generan necesidades educativas que el abordarlas solamente desde el sistema formal educativo sería insuficiente y no garantizaría el derecho.

El sistema educativo ciertamente, y por definición, en su dimensiones organizativas, docentes, metodológicas y de orientación, habrá de garantizar este derecho. Los educadores deberán tener muy en cuenta, asimismo, el derecho del alumno a la educación tanto en la dimensión de desarrollo individual como social. Por lo tanto, la teoría y la práctica educativa, que busque garantizar y desarrollar este derecho, no puede limitarse a la búsqueda de una eficacia en el rendimiento curricular, sino que ha de extenderse a la integralidad de la persona humana en todas su dimensiones.

La Ley Orgánica de 1/1990, de 3 de octubre, de Ordenación General del Sistema Educativo (L.O.G.S.E.) y numerosos reales decretos, órdenes ministeriales y otras espe- 
cificaciones oficiales, que desarrollan esta ley para el Sistema Fomal Educativo Español, facilitan a profesores, educadores y a toda la comunidad escolar gran riqueza de pautas y cauces operativos. Sin embargo, esta profusión legisladora serviría de muy poco para los menores con dificultades de socialización, si el reto queda reducido a los límites de la escuela, y dentro de ésta, a un problema curricular más.

Este planteamiento nos permite concluir que la acción para ayudar en el personal proceso de socialización a los sujetos y grupos, que por determinadas características innatas o adquiridas, o por encontrarse en situaciones sociales de desamparo, dificultad o conflicto social encuentran mayores dificultades en su proceso de socialización, supera los límites de lo estrictamente pedagógico y requiere una acción interdisciplinar. Por lo tanto, la complejidad y multifactorialidad de este problema no puede ni debe quedar restringida al ámbito escolar o al familiar, sino que requiere también la participación coordinada de numerosos agentes y agencias sociales extraescolares y extrafamiliares.

En este estudio, pretendo ofrecer pautas que ayuden a avanzar, aunque solo sea un pequeño paso, a la difícil tarea educativo-social, en la que están embarcados, desde hace años, tantas y tantas familias directamente afectadas, así como instituciones públicas y de iniciativa social, al tiempo que numerosos voluntarios y profesionales de la educación y de otras profesiones.

\section{Evolución del problema y situación actual.}

\subsection{Hitos principales en la evolución del problema.}

La percepción de los menores con problemas en el proceso de socialización ha estado condicionada en el transcurso histórico por dos situaciones principales y fuertemente relacionadas e influenciadas entre si. Esta son:

a) la corriente científico-teórica predominante en la explicación del fenómeno,

b) la actitud social ante los menores que presentaban dicha dificultad.

La historia nos muestra que los menores con dificultades de socialización no siempre han sido considerados como sujetos con derecho a educación, y, en algunos casos, ni siquiera como sujetos de derecho alguno (J. Merino, 1982 y 1985). El estudio realizado por Virginia Sabanza $(1995,4-116)$ sobre la educación especial a través de la historia ayudará a entender que el hecho de que hoy podamos hablar de la educación de los menores con dificultades en su proceso de socialización ha sido una conquista científica y social bastante larga y difícil.

Esta actitud social hacia los sujetos con deficiencias físicas o psíquicas y hacia los que entraban en conflicto con la sociedad ha evolucionado desde considerarlos como algo a eliminar en sus primeros momentos, hasta la actual que defiende la aceptación de los mismos como sujetos de todo derecho. Entre medio de estos dos extremos, sobresalieron dos actitudes y acciones principales. La primera se caracteriza por una crueldad rechazable desde cualquier ángulo. Me refiero a la predominante durante siglos que ocultaba desde su nacimiento a los nacidos con defectos porque se percibían como un castigo de Dios, fruto del pecado, y en consecuencia, 
una vergüenza para sus progenitores, al tiempo que despertaban en la sociedad sentimientos y actitudes negativas hacia ellos. La segunda actitud y modo de actuar es menos cruel pero también rechazable. Estamos hablando de la conocida tendencia a recluirlos en macro o microinstituciones lo más alejadas posible de los núcleos urbanos (F. Casas, 1988).

Diferentes concepciones teóricas del fenómeno se han desarrollado paralelamente a las actitudes y acciones sociales descritas. Estas concepciones van desde la explicación del fenómeno como un determinismo o cuasideterminismo biológico, psicológico o social hasta la tendencia, predominante en la actualidad, que entiende y explica las dificultades en la socialización como un proceso complejo de aprendizaje social, en el que los factores y procesos biológicos, psicológicos y sociales confluyen, en mayor o menor medida, según cada caso.

El siguiente esquema, en el que se recogen y sistematizan los principales hitos explicativos del fenómeno de la inadaptación, ayudará al lector a formarse una idea global del problema desde su perspectiva evolutiva:

\begin{tabular}{|c|c|c|}
\hline CRITERIO EXPLICATIVO & GÉNESIS & SISTEMA ORGANIZATIVO RESULTANTE \\
\hline BIOLÓGICO & $\begin{array}{c}\text { HERENCIA } \\
\text { (enfermedad) }\end{array}$ & $\begin{array}{c}\text { MACROINSTITUCIONES } \\
\text { (cárceles, asilos, hospitales } \\
\text { medievales, ...) }\end{array}$ \\
\hline PSICOLÓGICO & $\begin{array}{l}\text { CONFLICTO } \\
\text { SUBYACENTE } \\
\text { (enfermedad) }\end{array}$ & $\begin{array}{c}\text { AISLAMIENTO } \\
\text { (búsqueda de sistemas } \\
\text { alternativos...) }\end{array}$ \\
\hline SOCIOLÓGICO & $\begin{array}{l}\text { PRODUCTO } \\
\text { SOCIAL } \\
\text { terminismo social) }\end{array}$ & $\begin{array}{c}\text { INTERVENCIÓN } \\
\text { CONTEXTUAL } \\
\text { • progresiva } \\
\text { desinstitucionalización } \\
\text { - programas globales sec- } \\
\text { torializados de prevención } \\
\text { (detección, atención, seguimiento) }\end{array}$ \\
\hline
\end{tabular}

\begin{tabular}{ccc}
\hline INTERACCIONISTA & PROCESO & PREVENCIÓN, \\
DIALOGICO & DE APRENDIZAJE & - primaria \\
& $\begin{array}{c}\text { (ayuda educativa } \\
\text { y social) }\end{array}$ & $\bullet$ secundaria \\
& & terciaria \\
\hline
\end{tabular}

El análisis detenido del cuadro anterior ayudará a una comprensión más global del problema.

La columna de la izquierda refleja la evolución del criterio explicativo. En ella observamos el cambio sufrido por éste. Ha experimentado una auténtica transformación que va desde considerar como único criterio explicativo de las dificultades de socialización lo biológico, hasta la corriente actual defensora de una explicación más integral e interactiva, pasando por etapas intermedias que lo atribuían de manera 
exclusiva o predominante a lo psicológico o a lo social. En la explicación biológica, las dificultades, desviaciones o fracasos en la socialización se atribuyen a determinismo biológicos, cual es el caso de Lombroso cuando afirma que el criminal nace, no se hace; la psicológica a determinismos o cuasi determinismos psicológicos (Aichhorn, Klein, Redl, etc.), y la tercera a determinismo sociales. El criterio explicativo social ha adquirido gran impulso en las últimas décadas como paradigma de investigación y de acción. La última o interaccionista dialógica considera que el proceso de socialización es una actividad del sujeto en el que lo biológico, psicológico y social confluyen interactivamente. Esta interacción es activa de tal manera que las dificultades de socialización se deben generalmente al conjunto de los factores, sin que ello signifique que unas veces predomine lo biológico, otras lo psicológico y otras de lo social. Podemos decir, a tenor de la explicación interaccionista, que el inadaptado y delincuente se hace, no nace; aunque su potencial innato constituya un condicionante, posibilitante u obstaculizante, del proceso de socialización.

El modelo de análisis para estudiar e interpretar la génesis y desarrollo del problema a partir del criterio, predominante en cada momento, se refleja en la columna del centro. El criterio biológico y psicológico potenciaron la consideración del problema como una enfermedad, y por lo tanto el desarrollo de modelos clínicos de análisis, tanto en su diagnóstico como en la búsqueda de medidas de intervención.

La columna de la derecha sistematiza el modelo de intervención y el sistema organizacional resultante a partir del criterio y del modelo de análisis. Así el criterio biológico potenció los sistemas de intervención policiales, legales y médicos y las actitudes sociales e institucionales tendentes a eliminar a los sujetos con problemas o a su reclusión en macroinstituciones alejadas de las ciudades. Instituciones donde los menores se amontonaban con toda clase de adultos nada recomendables. El psicológico consiguió disminuir la dureza de las leyes y de las reclusiones y abrir un proceso de apertura y búsqueda de otras alternativas de intervención. El social potenció el desarrollo de métodos basados en la sociedad y en la comunidad. El interaccionista, aunque muy influenciado todavía por el lastre de etapas anteriores, se encuentra inmerso en la búsqueda de estrategias personales, institucionales y sociales centradas en la persona y en su contexto con el fin de individualizar, en la medida de lo posible, el tratamiento del problema, propulsando métodos comunitarios, psicológicos, compensatorios o educativos.

Sin embargo, tanto en el lenguaje político y de los medios de comunicación como en el profesional, existe la costumbre de llamar educativos o pedagógicos a todos estos métodos. Conviene salir al paso de esta indeterminación conceptual y precisar cuándo el tratamiento médico o psicológico, así como las numerosas acciones sociales asistenciales, económicas, instructivas o laborales, son educativas y cuándo no. En este trabajo ofreceré algunas orientaciones para facilitar una mayor precisión en este sentido.

La ubicación histórica de cada una de las explicaciones y modelos de intervención resultantes van de mayor antigüedad (biológico) a mayor actualidad (interaccionistadialógico).

Se deduce fácilmente del cuadro anterior que las explicaciones teóricas y el modelo de intervención derivado condicionaron no sólo la actitud social ante los menores con dificultades en la socialización, fuera cual fuere su causa o etiología, sino tam- 
bién la línea de investigación sobre el mismo. En la ponencia (J. Merino, 1992, 8799) que presenté en la Semana de Educación Social realizada en la Facultad de Educación -Centro de Formación de Profesorado- de la Universidad Complutense de Madrid (17 a 20 de noviembre de 1992) bajo el patrocionio del Ministerio de Asuntos Sociales, analizo las derivaciones teórico prácticas de cada uno de las tres corrientes explicativas y de los modelos teóricos de acción reflejados en el esquema.

Las explicaciones parciales $y$, por lo tanto, reduccionistas, y las acciones o intervenciones crueles, inadecuadas o insuficientes han pasado a la historia. Los menores que tienen dificultades en su proceso de socialización no se consideran ya como algo a ocultar y a encerrar en instituciones para aislarlos de la sociedad. Por el contrario, se buscan formas multidisciplinares de ayuda al considerarlos sujetos de todo derecho, pero que por su especificidad o peculiaridad generan necesidades educativas especiales.

\subsection{Menores con necesidades educativas especiales.}

El Real Decreto 696/1995, de 28 de abril, de ordenación de la educación de los alumnos con necesidades educativas especiales, se centra principalmente en "los alumnos con necesidades educativas especiales temporales o permanentes cuyo origen puede atribuirse, fundamentalmente a la historia educativa y escolar de los alumnos, a condiciones personales de sobredotación o a condiciones igualmente personales de discapacidad sensorial, motora o psíquica". Pero entiende igualmente que los alumnos con dificultades en su proceso de socialización, cuya etiología incluya también otros tipos de causas diferentes a las descritas, son asimismo alumnos con necesidades educativas especiales. Este Real Decreto se hace eco de la situación actual del tema, cuando, al presentar quiénes son los sujetos con necesidades educativas especiales, dice:

(...) su origen -las necesidades educativas especiales- puede atribuirse a diversas causas relacionadas fundamentalmente, con el contexto social o cultural, con la historia educativa y escolar de los alumnos o con condiciones personales asociadas bien a una sobredotación en cuanto a capacidades intelectuales, bien a una discapacidad psíquica, sensorial o motora o a trastornos graves de conducta.

Durante los últimos años, no es extraño encontrar en círculos teórico-científicos, profesionales y sociales, la idea de que la expresión "sujetos con necesidades educativas especiales" constituye una nueva terminología con mayor sensibilidad humana y social utilizada para substituir a la anterior de educación especial, pero que no implica cambios significativos para la acción. El Real decreto 696/1995 refleja que la expresión significa algo más que un simple cambio de terminología. Recoge toda una nueva concepción del fenómeno. Concepción que podemos resumir en los siguientes términos: los sujetos con necesidades educativas especiales son personas humanas normales, que por diferentes causas biológicas, psicológicas o sociales, tienen, de manera innata o adquirida, problemas para desarrollarse personal y socialmente en los sistemas standard de organización social y educativa. Consecuentemente, requie- 
ren una ayuda específica o especial para garantizar y desarrollar su derecho a la educación en igualdad de condiciones con el resto de los menores.

Este análisis nos ayuda a salir al paso de dos posturas sociales y profesionales incorrectas:

1. Circunscribir los menores que tienen problemas de socialización a los niños, adolescentes y jóvenes que muestran conductas inadecuadas o conflictivas. Me refiero a la expresión tradicional de menores y jóvenes inadaptados y delincuentes.

2. Reducir los sujetos con necesidades educativas especiales a los tradicionalmente considerados como sujetos de educación especial.

En este sentido, basados en la situación actual del tema, y siguiendo la misma línea argumental del Real Decreto citado, serán menores con dificultades en la socialización, aquellos que:

a) han tenido o tienen un contexto cultural deprivado, desfavorable, empobrecido $o$ inadecuado.

b) han tenido o tienen una situación social de desamparo, dificultad o conflicto social.

c) han tenido o tienen situaciones de riesgo, bien por desamparo, dificultad o conflicto familiar y social constante o porque han realizado o realizan conductas molestas y conflictivas para los demás miembros de la sociedad. Es obvio que estas situaciones de conflicto y de conductas desviadas no son el clima social más adecuado para una socialización positiva o si se quiere educativa, sino el cauce óptimo para iniciar y desarrollar procesos de inadaptación y delincuencia.

d) han tenido o tienen limitaciones biológicas, psicológicas o intelectuales, debidas a factores de naturaleza genética o hereditaria (Parálisis cerebral, Down, deficiencias y disfunciones mentales y psíquicas...), o resultado de factores o situaciones actuales (enfermedades permanentes o de larga duración, accidentes, desnutriciones, ingestión de drogas, fármacos, etc.).

e) han tenido o tienen una historia escolar inadecuada, insatisfactoria o negativa, tanto en lo que se refiere al rendimiento académico, como a las relaciones sociales con sus iguales y con los profesores en los centros educativos. Una y otra originan en los menores situaciones personales y relaciónales de gran conflicto consigo mismos y con los demás. Situaciones de dificultad o conflictos que no quedan entre las paredes de la escuela, sino que se extienden a la familia y a la calle. Este conflicto, dificultad, inadecuación e insatisfacción puede ser tanto por exceso (el problema de los superdotados), como por defecto (diferentes tipos de disfunciones, discapacidades y limitaciones). Este no es un problema de los últimos cinco años. Las situaciones dramáticas, a veces sin retroceso, en el proceso de socialización, a las que puede conducir un historial escolar negativo, fueron reflejadas magistralmente, ya en 1984, por Julio López de Oruezabal, Juez de Menores en el Tribunal Tutelar de Madrid, al prologar el libro de L. Martín Barroso (1984,8) titulado las fugas de menores. Prevención y tratamiento. Reproduzco textualmente unas líneas, porque su lectura ayuda a entender la magnitud de este factor: 
Sabemos por experiencia que tras la conducta profundamente perturbada y desorientada de un adolescente está siempre la huida de algo, de alguien, hacia alguna parte, sin brújula ni norte que le sitúe en su realidad. Pero lo sabía de manera global e imperfecta. (...).

Dice el autor -se refiere al autor del libro- que la fuga es "huida", "escape", "alejamiento", al fin, "derrota". Esta descripción-definición, aplicada al niño y adolescente, reviste caracteres dramáticos, porque supone haber llegado al límite de sufrimiento, a la tensión psíquica, angustiosa en una situación conflictual, según Fenichel, citado por el autor, con un nivel o índice bajo de tolerancia a la frustración. En todo caso es preferible a la "fuga simbólica", cuyo fenómeno mental de alejamiento sume al muchacho en simas de alienación, encerramiento sobre sí, que debiera ser más preocupante, de lo que al parecer es para enseñantes, profesores y padres.

De la situación escolar y de huida descrita a la realización de conductas delincuentes y por lo tanto a entrar en conflicto constante con la realidad social hay un paso muy pequeño.

Esta enumeración o clasificación de los menores que tienen dificultades en su proceso de socialización no debe inducirnos a considerar cada una de ellas como compartimentos estancos. Todas y cada una de las situaciones descritas se interaccionan e influyen mutuamente. Con más frecuencia de la deseada una situación conduce a la otra.

Por otra parte, el hecho de que la socialización sea un proceso complejo y unitario de cada persona, aunque condicionado positiva o negativamente por diferentes factores individuales, relacionales y sociales, puede llevarnos a interpretaciones erróneas a la hora de buscar modelos y formas de acción eficaces que sirvan para todos. La excesiva sectorialización de la acción preventiva, reeducativa o recuperadora ha conducido históricamente no solo a una falta de eficacia de métodos y técnicas sino también a reduccionismos en la conceptualización del problema. Así mismo, la generalización del fenómeno como si fuera igual para todos, lleva consigo el riesgo de extrapolar técnicas y métodos de intervención a situaciones personales y sociales muy diferentes. No es lo mismo una dificultad de socialización debida a deficiencias o limitaciones innatas, que otra cuya causa está en contextos culturales deprivados o en situaciones familiares o grupales de conflicto.

El principio de armonía e individualización ha de estar presente en la búsqueda de la acción pedagógica, y en consecuencia educativa, más adecuada a cada situación y caso.

Podemos concluir que la acción pedagógica con menores que tienen dificultades de socialización es un proceso multifactorial, en el que la ayuda del tipo que fuere (económica, asistencial, personal...), y la orientación, formación y tratamiento han de integrarse en la acción pedagógica, pues la educación no puede confundirse con el simple cultivo o desarrollo intelectual (instrucción), ni con el tratamiento médico o psicológico sino que abarca también otras dimensiones de socialización del individuo que se desarrollan en la interacción con el medio cultural y social, al tiempo que requiere unas características específicas para que sean educativa. Esta sensibilidad impregna felizmente el Real Decreto 299/1996 de 28 de febrero, de ordenación de las acciones dirigidas a la compensación de desigualdades en educación, y de la mayo- 
ría de las regulaciones jurídicas sobre el mismo problema que han ido surgiendo en las diferentes autonomías del Estado Español.

\section{La acción pedagógica.}

\section{1. ¿Qué entendemos por acción pedagógica?}

La pedagogía es el arte, la ciencia y la tecnología de la educación. Por lo tanto la acción pedagógica es la acción que regula y dirige la educación.

Sin embargo, el problema no es tan simple como pudiera derivarse de una rápida lectura del párrafo anterior. La complejidad y conflictividad implícita en la acción pedagógica podemos sistematizarla en dos cauces principales. El primero viene determinado por la dificultad para concretar el concepto de educación. El segundo, porque en la acción pedagógica confluyen numerosos elementos antropológicos, sociales, personales, conceptuales y metodológicos, así como factores y procesos diferentes.

La educación como precisa y muestra J. Ma Quintana $(1995,47 \mathrm{ss})$ es un concepto polisémico. Este autor compara la educación con un poliedro que tiene muchas caras. Después de identificar y describir muchas de esas caras, J.M $M^{a}$ Quintana constata que no todas las caras las estudia la pedagogía. Por ejemplo, la educación como hecho y fenómeno social es objeto de la sociología, como factor económico es objeto de la economía de la educación, etc. Esto no quiere decir que la pedagogía excluya estas caras de la educación. No solo no las excluye, sino que las necesita para abordar la cara más interesante de la pedagogía, y que determinará su objeto. Este no es otro que el acto de educar y por lo tanto la acción de educar. Fundamentado en los argumentos que configuran este discurso, José $M^{a}$ Quintana concluye que la Pedagogía se preocupa principalmente de la acción educadora, mientras que para el conjunto de todas las ciencias que estudian las otras caras de la educación como factum y fenómeno social reserva la tan traída y llevada expresión en las últimas décadas de "Ciencias de la Educación" (ib.48). Comparto y asumo estas precisiones.

Esta polisemia aparece tanto en el nivel conceptual como en el operativo. La pedagogía ha abordado amplia y detenidamente ambos niveles.

Las numerosas definiciones, revisiones y sistematizaciones de la educación permiten aportar las siguientes características que nos orientarán en el plano conceptual a la hora de advertir y matizar si una acción es educación o no. Reproduzco textualmente las notas o características más repetidas en las definiciones de educación (J. Merino 1996,171-172):

- Categoría específicamente humana.

- Perfeccionamiento optimizante.

- Formación global e integral del hombre.

- Acción y proceso intencional.

- Preparar para la vida. Preparación condicionada por el contexto real en el que se vive.

- Reproducción de modelos antropológicos, culturales y sociales 
- Transmisión cultural, científica y tecnológica.

- Proporcionar habilidades para la vida en todas sus dimensiones.

- Aunque predomina la acción educadora del hombre sobre el hombre, sin embargo la naturaleza y las cosas también educan.

Conviene precisar que algunas de las características o notas indicadas pueden no ser educación, si se consideran fuera del conjunto.

Afirmar que la acción educativa se construye y desarrolla por la integración simultánea de dos polos, uno exterior al educando y otro interior al mismo, es una constante en pedagogía tanto a nivel teórico como operativo. El estudio de la acción educativa ha sido y sigue siendo una de las tareas más permanentes en la reflexión e investigación pedagógica (G. H. Wrigh. 1968 y 1979; J. Castañé, 1980 y 1981; J.L. Catillejo Brull, 1984 y 85; J. Trilla, 1985; J. V. Merino, 1987; G. Jover, 1991; J. Ma Quintana, 1995). De estos y otros muchos estudios, no referidos aquí, se derivan dos conclusiones principales:

- La acción educativa es una acción intencional perfectiva que incluye los dos polos indicados, acción del educador y acción del educando.

- No hay educación sino se produce el efecto de educar.

Estos dos polos, acción exterior al educando o acción del educador y acción del propio educando, pueden considerarse separadas en los análisis teóricos del fenómeno, y en algunos casos, discurrir por caminos distintos en la práctica. Pero no habrá educación si ambas acciones no confluyen en un único proceso de acción, proceso que se denomina "acción educativa". Conviene insistir que para que haya educación se requiere, además de lo anterior, que se consiga el efecto de la acción.

La acción pedagógica en el marco de este discurso, sería la acción educativa regulada científica y tecnológicamente.

3.2. Características específicas de la acción pedagógica con menores que tienen dificultades de socialización.

\subsubsection{Unidad de acción interdisciplinaria.}

El esfuerzo por delimitar la especificidad de cada acción educativa que respondía a diferentes ámbitos, ha abierto en ocasiones debates estériles que, en mi opinión, originaban antinomias u oposiciones artificiales. En nuestro caso concreto, hay que subrayar aquellas tendencias teóricas y prácticas que oponen la "educación escolar" a la "educación social", como si una y otra constituyeran compartimentos estancos o tipos distintos de educación que contrarios y enfrentados entre sí. No ponemos ningún reparo al planteamiento de la antinomia como tal antinomia pedagógica. Sin embargo, el hecho de que cada uno de los polos de la antinomia ponga el acento en dimensiones de la persona distintas, no autoriza a considerarlos como tipos de educación diferentes y opuestos.

Acudir a los dos principios pedagógicos clásicos siguientes ayudará a resolver esta antinomia: 
1. El principio de unidad y diferenciación del proceso educativo,

2. Las antinomias pedagógicas no son polos opuestos, cual es el caso de las antinomias lógicas, sino complementarios.

El segundo principio ayuda a matizar si una acción es educativa o no. El hecho de que en un determinado polo destaque una dimensión o proceso concreto de la educación, no significa que el polo opuesto se excluya, sino que el primero ejerce un papel predominante en el proceso o acción educativa concreta, pero necesita al otro como complemento para que se dé educación. Si así no fuera, es posible que estuviéramos hablando de otra cosa, pero no de educación. Por ejemplo, la enseñanza, imperante en el polo "educación escolar", puede ser educación o no. En este mismo sentido, la ayuda comunitaria al menor en dificultad social, que prevalece hoy en el polo "educación social", puede ser educación, pero también puede no serlo.

La acción pedagógica, en nuestro caso, que a partir de ahora denominaremos acción pedagógico-social, no debe identificarse solamente con la educación social. Predominará el polo de educación social en unos casos, y el de educación escolar, en otros. Por ejemplo, de entre los diferentes grupos de menores con dificultades de socialización que delimitamos anteriormente, los tres primeros (contexto cultural deprivado..., situación social de desamparo o dificultad social..., y situaciones de riesgo) requerirán una mayor incidencia del polo educación social; mientras que aquellos que deben sus dificultades a limitaciones o deficiencias hereditarias o a resultado de situaciones traumáticas -grupos d) y e)- requerirán un impulso mayor de la educación escolar.

Lo anterior nos permite recuperar e incluso reivindicar el principio de unidad, diferenciación e integralidad como primer principio normativo de la acción pedagógicosocial, y que formuló en los siguientes términos: la acción pedagógico-social con menores que tienen dificultades en su socialización ha de integrar todos los procesos, ámbitos y acciones en un una unidad de acción que tenga como eje integrador las exigencias de la educación, $y$, en consecuencia, el perfil personal y social del sujeto educando y los condicionamientos personales y socioculturales del mismo.

\subsubsection{La prevención es nota inherente de la acción pedagógico-social.}

La acción pedagógica por su naturaleza educativa es fundamentalmente preventiva y sólo de manera subsidiaria y complementaria es correctiva. El término preventivo lo entendemos aquí como el proceso capaz de adelantarse a aquellas situaciones y acciones que dificultarán, desviarán o paralizarán el proceso de aprendizaje social de los sujetos y grupos. Con esta anticipación trata de impedir que se den las dificultades en la socialización.

El concepto de educación, por definición, lleva implícito el de prevención. En este contexto, y en el caso concreto que ahora estudiamos, podemos definir la acción pedagógico-social, como el conjunto de acciones y omisiones, sociales o académicas, que ayudan al educando a progresar positivamente en su proceso de socialización, procurando que la dificultad y conflicto social no se origine (prevención primaria) o no se desarrolle (prevención secundaria y terciaria). 
El hecho de que la prevención primaria, conceptual y operativamente, esté menos determinada que la secundaria y terciaria lleva consigo el riesgo de reducir la acción pedagógico-social a prevención secundaria y terciaria. Conviene estar en guardia frente a este riesgo para no reducir la pedagogía social a tratamientos o metodologías correctivas de reeducación y resocialización. La pedagogía social no excluye las acciones reeducativas $y$ resocializantes, pero ha de ser fundamentalmente, como antes indicamos, acción de prevención primaria. Acudir al ejemplo de la educación como acción orientada a erradicar el analfabetimo ayudará a explicitar más esta idea. ¿qué pensaríamos de una sociedad o de un sistema educativo que no pone los medios para evitar el analfabetismo, con el fin de hacer posteriormente alarde de unos métodos excelentes de desalfabetización?. Considero que el mismo discurso es válido en la acción pedagógico-social. El esfuerzo mayor hay que realizarlo en la prevención primaria, en adelantarse al surgimiento del riesgo y del problema, y sólo en el caso de que esta acción preventivo-educativa no haya sido exitosa, acudir a la secundaria y terciaria.

No quisiera que del análisis anterior se extrajera la falsa conclusión de que hay que abandonar la secundaria y terciaria. Todo lo contrario, puesto que primaria, secundaria y terciaria no son tipos de ayuda o procesos educativos diferentes y excluyentes, sino fases de un mismo proceso (J. Merino, 1987, 180-185). Por lo tanto, a nivel de investigación y de acción hay que desarrollar las tres.

\subsubsection{Acción humana y tecnología.}

La búsqueda de métodos eficaces en la prevención y tratamiento de las dificultades y conflictos en el proceso de socialización ha puesto en peligro o al menos viciado durante las últimas décadas una de las características de la acción pedagógica. Me refiero a la relación humana que toda relación educativa implica. La importancia y urgencia del problema se manifiesta en el hecho de que numerosos autores hayan retomado como objeto de estudio prioritario el clásico tema de la relación educativa y la comunicación entre profesor y alumno en el proceso de enseñanza y aprendizaje. La importancia de la relación humana en la enseñanza-aprendizaje y en la acción tutorial se convierte en una constante no sólo cuantitativa, por el hecho de su reiteración, sino también cualitativa por el alcance que se le atribuye y la profundidad con la que se estudia en los últimos años (Albinum, J. 1977; Colom, T. 1982; Fernández, P. 1991; Franta, H. 1982; Ibañez-Martín, J. A. 1987; JOVER, G. 1991; LÁZARO, A. 1986). La importancia de la relación humana como factor clave en la creación y desarrollo del clima social adecuado para el mejor desarrollo de la acción educativa se aplica no solo a la educación social, sino también al ámbito didáctico. La tarea de crear climas sociales positivos en el aula y en los centros educativos ha adquirido un gran impulso en la última década (BALL, S.J. 1989; MEDINA, A. 1993).

La relación entre educadores y menores que tienen problemas en el proceso de socialización requiere con mucha mayor razón esa dimensión humana de la comunicación educativa.

Lo anterior no significa ni quiere ser una crítica velada al cultivo y desarrollo de técnicas y metodologías eficaces de tipo clínico, psicológico, comunitario, didáctico, 
legal, policial o de otro tipo, sino que su eficacia será muy baja en la mayoría de los casos, como nos muestra la historia, si no va acompañada de una relación humana de aceptación y comprensión, de un cultivo de valores personales y sociales positivos o de climas sociales de aceptación y no de exclusión.

Lo técnico sin lo humano corre el riesgo en prevención (primaria, secundaria o terciaria) de construir y aplicar rosarios de test, entrevistas técnicas, programas de desarrollo individual técnicamente bien hechos desde la perspectiva experimental, didáctica, psicológica, tutorial, clínica, etc., pero conducir a un objetivo distinto al pretendido. Así, en lugar de constituir una ayuda para el menor, derive hacia el efecto contrario de etiquetarlo con las consecuencias negativas para su autoestima o para la aceptación de sus compañeros y profesores.

Numerosos educadores, reunidos en la Semana de Educación Social (Muñoz, A. 1992) constataron otra subversión del siguiente principio clásico en pedagógia: el método y la técnica son instrumentos para y al servicio del educando. Esta subversión consistía, según los educadores, en que la normal y loable búsqueda de una eficacia técnica cada vez más objetiva y generalizable ha conducido, con más frecuencia de la deseada, a que la técnica pase de instrumento a fin en sí misma, con el riesgo de que el menor acabe estando en función de la técnica y no viceversa.

Sin abandonar ni bajar el ritmo de investigación y experimentación de métodos y técnicas cada vez más eficaces, hay que potenciar las relaciones humanas e incrementar el desarrollo de climas sociales positivos en los que el menor con dificultades o conflictos en su proceso de socialización se encuentre aceptado y motivado para avanzar en su socialización, o corregirlo en su caso. Hay que ir a planteamientos globalizadores e integradores que posibiliten y potencien el desarrollo de una pedagogía de la inclusión y no de la exclusión.

\subsubsection{Acción personalizada.}

La influencia del positivismo y de los paradigmas de investigación de las ciencias naturales en la investigación de las ciencias humanas y sociales condujo durante gran parte de este siglo a un predominio de lo nomotético como paradigma de investigación e intervención en la acción pedagógica. Durante los últimos años, lo idiográfico ha ido abriéndose paso, de tal manera que la acción pedagógica se caracteriza hoy por una mayor personalización. Esto requiere un esfuerzo para que los programas y acciones de intervención educativa sean cada vez más individualizados.

El educando y su contexto más próximo con sus necesidades educativas especiales se convierte en el criterio y eje principal sobre el que ha de construirse y desarrollarse la acción pedagógico-social. Es el diagnóstico del mismo, con la ayuda de los resultados de investigaciones experimentales, quien determina, o al menos condiciona en gran manera, el modelo y la metodología de intervención. Esta no es un proceso deductivo a partir de resultados de investigaciones que se generalizan y a veces extrapolan a situaciones personales y sociales diferentes, sino un proceso individualizado. No es extraño que hoy se insista más en la intervención educativa individual y grupal, que en la aplicación aséptica y neutramente objetiva de técnicas y estrategias generales. 
La insistencia en la personalización lleva consigo también la desmitificación de determinadas formas institucionalizadas consideradas durante siglos como únicos cauces válidos de la acción pedagógico-social, cual es el caso de la escuela y de las instituciones sociales y asistenciales más o menos formales. Tanto a nivel de investigación como de acción, se caricaturiza hoy a estas corrientes generales anteponiendo el prefijo "des" a aquellas formas de actuar tradicionales, por ejemplo, desinstitucionalización, desmedicalización, despsicologización, desjudicialización, etc. En este contexto, la individualización del diagnóstico y de la intervención predomina en la actualidad sobre la aplicación, más o menos generalizada y extrapolada de programas estándar. Ello lleva consigo la aceptación en el ámbito teórico y práctico de una acción pedagógico social interdisciplinar y personalizada.

Esta concepción personalizada, integral e interdisciplinar difícilmente podrá progresar y hacerse operativa si no va acompañada de un marco social con estructuras y capacidad suficiente para tolerar y admitir la pluralidad y la diferenciación. La investigación social, psicológica y pedagógica deberán caminar también en esta línea multi e interdisciplinar. Los estudios actuales del desarrollo del niño y la intervención psicopedagógica sobre las dificultades del desarrollo confirman esta exigencia y constituyen una base argumental válida al respecto (M. Moreno, 1997). Las investigaciones sobre la agresividad van confirmando asimismo cada vez más que ésta es fruto de la interacción de factores individuales, innatos o adquiridos, y de agentes y situaciones sociales ( A. P. Goldstein, 1991; Foro Europeo de Seguridad Urbana, 1993; J. Martín Ramírez, 1993, 1996 y 1997; N. P. STROMQUIST, 1996).

Por otra parte, las orientaciones sobre la investigación social insisten cada vez con mayor reiteración en la necesidad de que investigación e intervención caminen unidas.

Con cierta frecuencia ha existido una desconexión entre la exigencia de interconexión requerida por la integralidad de los fenómenos sociales y educativos, y los planteamientos de análisis que, anclados en el atomismo y positivismo experimental, se empeñaban en mantener ciertos reduccionismos clásicos. Integralidad propugnada implícitamente por los mismos investigadores sectoriales cuando intentan aplicar el resultado de su análisis en los modelos de intervención. Dichos modelos, al verse obligados, por razón de eficacia, a estar más inmersos en la realidad personal y social de los educandos con dificultades en el proceso de socialización, descalificaban indirectamente la sectorialización del análisis. Esta realidad ha contribuido a poner de manifiesto los reduccionismos existentes en la tendencia atomista, al tiempo que confirman la hipótesis de que investigación e intervención forman una unidad de acción interrelacionada e interinfluyente en la integralidad del problema. En este contexto, J. Ortega Esteban (1994) denuncia ciertas incoherencias y errores existentes entre la investigación de los problemas de inadaptación y delincuencia y la intervención sobre el mismo.

A mi entender, errores de enfoque y planteamiento de los análisis del problema, discordancia con la realidad, algunos sesgos de oficio (psicologización y sociologización) y, en algún caso, tufos ideológicos han influido en los planteamientos y en los resultados. En efecto, en general, esas investigaciones -se refiere a investigaciones sectoriales o enmarcadas en niveles diferentes al de la intervención- se han dirigido al análisis de los individuos y no al de la comunidad en la que viven los individuos. Si pretendemos implementar modelos, pla- 
nes, programas o proyectos comunitarios, lógicamente los estudios de análisis o diagnóstico, se puede hacer un análisis o diagnóstico previos, deben participar de la misma lógico comunitaria. No se puede hacer un análisis individual y luego implementar un programa comunitario. Los planteamientos y diseños de los "análisis" deben ser integradores, interrelacionales y comunitarios, con metodologías analíticas y experimentales, pero sin perder la visión unitaria e integrada del conjunto. Los planteamientos estrictamente analíticos, al desintegrar y aislar los factores etiológicos de la delincuencia, nos despistan de la genética de las causas y dispersan y difuminan las responsabilidades a compartir por parte del colectivo social y a su vez nos alejan de la realidad sistemática, conexionada y dinámica de todos los procesos sociales.

La personalización no significa, por lo tanto, desconexión de la propia realidad social en la que los individuos van configurando o construyendo su personalidad, sino integración de todos los factores (individuales y sociales) en torno al proceso de persona-génesis (J.M Quintana Cabanas 1984) del educando y por consiguiente a su proceso de socialización.

\section{Del análisis a la práctica.}

La teoría desconectada de la práctica se descontextualiza y separa cada vez más de la realidad concreta. La práctica sin la teoría se dispersa y camina con frecuencia dando bandazos estériles. El análisis anterior derivaría en pura teoría especulativa si no tuvieran una incidencia real en la práctica pedagógico-social del día a día. En este apartado sistematizaré una serie de principios y orientaciones con la única intención de aportar mi granito de arena a la difícil tarea de unir teoría y práctica.

\subsection{Principios generales inspiradores de la práctica.}

-Principio de unidad y diferenciación. La acción pedagógico-social tiene una finalidad educativa porque es el arte, la ciencia y la técnica de la educación social. La educación es un proceso unitario y diferencial.

Las dos afirmaciones anteriores que emanan del principio se han convertido en planteamientos casi axiomáticos en la reflexión pedagógica y llevan consigo una exigencia para la configuración y desarrollo de la acción pedagógico-social. Esta no puede ser la suma de actividades puntuales, dispersas e incoherentes entre sí, ni tampoco un constructo puramente funcional que se edifique exclusivamente con y a partir de las exigencias demandadas en cada momento por la realidad personal y social. Por supuesto que las respuestas funcionales son elementos constitutivos de la acción pedagógica, pero integradas y activamente interdependientes en ef sistema unitario que la educación, como "desarrollo intencional perfectivo" (Facultad de Filosofía y Ciencias de la Educación, 1984,205), construye y configura.

El riesgo de que la intervención sobre los menores con dificultades en el proceso de socialización derive en un mosaico de acciones inconexas e incorrectamente denominadas pedagógicas o educativas es muy alto, ya que la acción pedagógico- 
social ha de responder, por supuesto, a las diferentes dimensiones de la persona y a las distintas situaciones contextuales y personales en las que el educando pueda encontrarse, con el fin de fortalecer aquellas potencialidades y situaciones posibilitantes y favorecedoras de la socialización, y controlar, neutralizar o regular las que lo obstaculizan. Esta dinámica hace que en la acción pedagógico-social confluyan numerosos agentes, elementos, factores y procesos de origen y configuración desigual, y, a veces, contradictorios, convirtiéndola en una acción multipolar e interdisciplinar.

Este principio inspira y condiciona el desarrollo de la acción educadora de tal manera que las respuestas concretas a las diferentes dimensiones y situaciones dispares de la práctica educativa se configuren, interaccionen y desarrollen en el marco unitario del proceso educativo. Los principios siguientes ayudarán a concretar las exigencias operativas de este principio.

-Principio de integralidad. Este principio tiene dos exigencias prácticas. Por una parte, la acción pedagógico-social ha de constituir una respuesta a la totalidad integral del problema, sea de manera globalizada o interdisciplinar. Si responde solamente a alguno de los sectores o procesos del problema será, como antes indicamos, otro tipo de acción (médica, psicológica, económica, policial, manipuladora, comunitaria, política, asistencial...) pero no pedagógica, y en consecuencia, no necesariamente educativa. Por otra, ha de ser integradora, esto es, poseer la suficiente capacidad teórica y práctica para integrar en una unidad de acción educativa todas y cada una de las variables, factores agentes y procesos que intervengan en la acción.

-Principio de realismo. Toda acción humana ha de intentar ser realista y, por supuesto, también la acción educativa. En el caso concreto que nos ocupa, hablamos de necesidades educativas especiales en el proceso de educativo de los educados que tienen problemas de socialización. Nótese que estamos utilizando el término educando, de manera indistinta con el de menor en el desarrollo de este trabajo. Con ello queremos insistir en que el menor que tiene dificultades en el proceso de socialización es un educando con necesidades educativas especiales que necesita una ayuda específica, pero no un individuo a rechazar, ocultar o a etiquetar como algo raro. Conviene en este sentido no dejarse llevar por utopías -valga la redundancia- excesivamente utópicas, teorías científicas o concepciones del hombre y de la sociedad muy bonitas pero inviables, determinismos generados por intransigencias y fanatismos ideológicos, religiosos, económicos, por actitudes personales y grupales excesivamente etnocentricas, o por mecanismos de defensa del estatus social conscientes o inconscientes.

-Principio de individualización personalizada. Sin pretensión de un existencialismo absoluto, me permito afirmar que de alguna manera el individuo humano es el constructor de su propia persona. El desarrollo personal y social es una tarea del individuo en interacción con el medio. El método socrático constituye uno de los mejores ejemplos de aplicación de este principio. Son muchos los filósofos que en el discurrir histórico reflejan esta idea que podemos resumir en las siguientes frases: "sé tú mismo", "el hombre es el mejor constructor de sí mismo", "constrúyete a ti mismo" o "el hombre es el agente de su propio destino".

-Principio de interdisciplinaridad. Los agentes, profesionales o no profesionales que pueden intervenir en la acción pedagógico-social constituyen un grupo numero- 
so. Desde la relación personal hasta el tratamiento más técnico, pasando por la familia y el grupo de iguales, existe un gran abanico de agentes y agencias que pueden participar de manera directa o indirecta. Conviene superar la simple multidisciplinaridad de profesionales y acciones inconexas entre sí en pro de una unidad de acción interdisciplinaria.

Los programas de desarrollo individual son un ejemplo de acciones interdisciplinarias. El desarrollo de este principio requiere a los educadores formar parte de equipos interdisciplinarios.

- Principio de contextualización. El educando forma parte de un entorno familiar, social y cultural determinado. No es pura biología, ni tampoco producto de un determinismo social. Este principio requiere operativamente que la acción pedagógicosocial mantenga al educando en su propio medio o al menos vinculado al mismo, a no ser que este medio constituya un factor completamente nocivo para su desarrollo personal y social.

- Principio de optimización. La educación tiene como finalidad última lograr el máximo de perfección en sus dimensiones individuales y sociales. La idea de optimización adquiere aquí el doble sentido de optimización de las posibilidades del propio individuo como tal en su proceso educativo y la optimización de los agentes, medios y recursos en orden a obtener el máximo rendimiento

- Principio de comunicación. La comunicación que conduzca a una relación humana entre educadores y educandos es la base de toda acción educativa. Es importante fortalecer cauces que posibiliten una comunicación directa y sincera entre educadores y educandos, sean aquellos profesionales o no. Ello no implica falsas renuncias a roles determinados. El educador no puede dejar de ser joven o adulto y convertirse en un niño. Pero sí puede generar actitudes y relaciones de diálogo, de entendimiento e incluso de amistad, en donde el educando se sienta seguro y encuentre situaciones de afecto y comprensión que le ayuden tanto a conocerse a sí mismo con sus posibilidades y limitaciones, como a comunicarse y relacionarse de manera normalizada con el medio. Ello le facilitará una respuesta adecuada al reto de su socialización. La acción pedagógica es fundamentalmente comunicación y las diferentes estrategias, metodologías y técnicas no son sino instrumentos para favorecer dicha comunicación.

- Principio de aceptación. El menor es una persona con sus peculiaridades diferenciales. El hecho de que en muchos casos, estas diferencias se alejen de la media, no justifica ninguna acción que considere al educando como un sujeto u objeto de experimentación. Insistí más arriba en la importancia de las relaciones humanas en la acción pedagógico social. Cualquier tecnología se mostrará insuficiente si no va acompañada de la aceptación del educando.

4.2. Principios de la acción pedagógico social en las fases de prevención primaria y secundaria.

Además de los principios generales descritos hasta ahora, las fases de prevención primaria y secundaria inspiran y regulan su acción a partir de otros principios específicos. 
Con relación a la fase de prevención primaria conviene recordar el dicho popular referido a las medicinas: "la mejor medicina es la que no se toma". Esta misma filosofía de la sabiduría popular debe impregnar la acción pedagógica en la fase de prevención primaria. Suelen considerarse como principios de esta fase (J. Merino, 1993,202-206) los siguientes:

-Principio de anticipación. Como su mismo nombre indica, anticiparse al problema, prever las limitaciones y dificultades en los que éste surge y evitar, o al menos dificultar, que el mismo se origine en el individuo o en la sociedad es la exigencia de este principio. Dos bloques principales constituyen su eje operativo.

1. Acciones centradas en el individuo y en sus procesos relaciónales. Las ayudas sociales y formativo-educativas se dirigirán no sólo hacia los educandos sobre los que se prevea la génesis y desarrollo de dificultades, sino también hacia quienes traten con ellos en la relación diaria y en la escuela. Esta acción preventiva se anticiparía al surgimiento de muchas de las dificultades de socialización. Cuantas se hubieran evitado si padres y profesores hubieran dispuesto de formación y de medios para adelantarse al problema o detectar los indicadores de que éste se estaba gestando.

2. Acciones sobre el medio. Desplegar políticas y acciones educativas, económicas, culturales, jurídicas, policiales, etc., con el fin de anticiparse a que nazcan $\mathrm{u}$ originen determinadas situaciones y contextos que se han mostrado constantemente como generadoras de dificultades en la socialización se percibe como acción preventiva importante. Numerosas situaciones de riesgo pudieran haber sido evitadas o neutralizadas si se desarrollaran programas de sensibilización sobre determinados procesos. Por ejemplo, educar para el consumo crítico y selectivo, diseñar y ejecutar programas formativos para la utilización positiva y creativa del tiempo libre, poner en marcha políticas económicas y sociales justas, posibilitar el acceso a los bienes culturales en igualdad de condiciones, construir instalaciones deportivas en el centro de las ciudades junto a las viviendas y colegios que faciliten el deporte de los menores y jóvenes sin necesidad de verse obligados a acudir a clubs específicos y generalmente caros, etc.

-Principio de normalización. Este principio no debe interpretarse en función de la media estándar sino de las características diferenciadoras de cada educando. Normalizar no quiere decir regular todo el proceso formativo para acomodar al menor a las exigencias profesionales y sociales de la media social, sino buscar, por una parte, el máximo desarrollo de las posibilidades de cada individuo, y por otra, compensar o corregir la realidad social para adecuarla en la medida de los posible a dichas posibilidades y limitaciones, con el fin de que los individuos y grupos se enfrenten en igualdad de condiciones a las exigencias de la realidad social.

Los menores con necesidades educativas especiales, sea cual fuere su procedencia, suelen encontrar mayores dificultades que otros para acometer en igualdad de condiciones su proceso de socialización. De ahí que el principio de normalización ha de buscar una armonía entre lo personal y lo social. Por lo tanto, no puede reducirse su actuación solamente al individuo, desarrollando al máximo las capacidades y posibilidades individuales, sino que ha de realizar también un esfuerzo para adecuar el medio a las limitaciones de algunos individuos y grupos. Recordar el pro- 
blema de las barreras arquitectónicas ayudará a comprender las exigencias operativas de este principio.

-Principio de ajuste. Es continuación y exigencia clara del principio de normalización. En la sociedad actual existen demasiadas situaciones que generan desajustes entre la capacidad asimilativa de los individuos y de los grupos y los mensajes recibidos a través de los medios de comunicación de masas. Por ejemplo, las sorprendentes, fantásticas y mágicas realidades sociales, presentadas por el marketing y la publicidad de la sociedad de consumo, despiertan artificialmente un nivel de aspiraciones desproporcionadas tanto a las posibilidades de logro de la mayoría de individuos y grupos, como a los cauces existentes en la sociedad para conseguir satisfacer dichas necesidades y aspiraciones por medios normales. Esta desproporción se incrementa en los menores con dificultades y limitaciones individuales o sociales. En este contexto, el desajuste entre aspiraciones y posibilidades es una constante en los menores que tienen dificultades de socialización. Intentar satisfacer su nivel de aspiraciones o conseguir las realidades sociales de la sociedad de consumo por medios poco normales es una tentación muy fuerte para muchos menores y de manera especial para los limitados o deprivados.

Dotar a los menores de actitudes, recursos de conocimiento y habilidades sociales para hacer frente a la agresión proveniente de los medios masivos de comunicación es una de las tareas principales de la acción pedagógico-social derivada de este principio. La familia y la escuela están llamadas a realizar una gran labor en este sentido.

Con relación a la fase de prevención secundaria conviene, además de recordar la mayoría de los principios anteriores, realizar ciertas matizaciones a algunos principios descritos en la fase primaria y a nivel general de la acción pedagógicosocial.

-Principio de precocidad. La prevención secundaria se caracteriza porque concentra su acción en aquellas situaciones personales y sociales percibidas, valoradas y experimentadas como de riesgo.

El sentido común y las más elementales orientaciones metodológicas de actuación sobre menores en riesgo insisten en que, cuanto antes se inicie el proceso de intervención, el riesgo de que la dificultad avance será menor. Por lo tanto, cuanto antes se neutralice la dificultad, se compense la carencia o se interrumpa el proceso desviado de socialización ya iniciado, más fácil será conseguir éxito en su reeducación y reinserción.

-Principio de contextualización. Casi todos los estudios sobre las dificultades en el proceso de socialización constatan que éstas son un producto de un desajuste, desviación o conflicto en la relación entre persona y medio. Fundamentar y desarrollar la acción pedagógico social en la propia realidad social donde la dificultad surgió se presenta como un principio clave de la acción.

Los principios de optimización, ajuste y realismo adquieren en esta fase una concreción mayor debido a que la situación de riesgo ya existe y suele ser más concreta que en la prevención primaria. 


\section{Dos instrumentos para la personalización de la intervención}

\subsection{Las adaptaciones curriculares individuales.}

La flexibilidad curricular dentro del sistema formal educativo es una de las exigencias de là atención a las necesidades educativas especiales. Son numerosas los esfuerzos que educadores (profesores, tutores...) están realizando para hacer operativa la flexibilidad curricular. Disponer de guías o instrumentos facilitará esta tarea. Desde hace años se trabaja en este sentido. En mi trabajo sobre "acción pedagógico preventiva de menores en desamparo y conflicto social" (J. Merino, 1996, 191-192), después de analizar las aportaciones de la LOGSE a este problema, presento un esquema guía para el registro de la adaptación curricular individual (A.C.I.) que estructuro y desarrollo sobre los siete bloques siguientes:

1. Identificación del alumno.

2. Profesionales participantes en la elaboración del A.C.I.

3. Identificación del documento.

4. Recogida de información relevante sobre el alumno para la toma de decisiones,

5. Toma de decisiones sobre las adaptaciones curriculares.

6. Criterios de promoción.

6. Seguimiento del A.C.I.

La remisión a dicho trabajo me libera de una explicitación mayor, puesto que algunos de los interrogantes que este esquema despierte podrán encontrar respuesta en el mismo.

\subsection{El estudio de caso como modelo de intervención.}

El estudio de caso está adquiriendo cada vez más fuerza en los procesos de planificación y de manera especial como modelo de intervención personalizada, puesto que análisis, diagnóstico e intervención constituyen una unidad de acción en el mismo. Presento a continuación una serie de orientaciones o, si se prefiere, un esquema-guía de los pasos a seguir para elaborar un constructo teórico práctico que facilite la toma de decisiones en la planificación y programación de la intervención social partir del estudio de caso. Este equema-guía sólo pretende proporcionar al lector un documento de trabajo base que le ayude a elaborar su propio constructo teórico-práctico.

Primer paso: evaluación inicial para determinar la génesis y desarrollo del caso en orden a establecer el plan de acción.

1. Recogida del máximo de información al respecto:

1.1. Descripción inicial del caso:

- Si es una persona, es prescriptivo recoger el máximo de información sobre la misma. Los instrumentos y cauces más usados para esta tarea son:

- autoinformes,(observación, entrevistas, diálogos, test, etc.). 
- heteroinformes (informes psicológicos, laborales, formativos, sociales, judiciales, escolares, (ej. ficha del educando: historial sanitario, académico, escolar; mandamiento de ingreso, etc.).

- marco situacional y jurídico en el que ha de resolverse.

Si es una situación, la recogida del máximo de información se podrá realizar a través de:

- investigación social en el sentido tradicional.

- consulta social.

- Análisis de la situación.

1.2. Marco teórico de referencia:

- estudios teóricos relacionados

- situaciones similares.

Paso segundo: diagnóstico

2.1. Análisis de toda la información anterior.

2.2. Búsqueda y/o elaboración de instrumentos, estrategias y métodos de diagnóstico ad hoc, así como la aplicación de los mismos para la elaboración de aquel.

2.3. Informe diagnóstico.

Paso tercero: delimitación y formulación de objetivos

- Generales.

- Específicos.

- A corto plazo.

- A medio plazo.

- A largo plazo.

Paso cuarto: plan de acción.

4.1. Especificación de programa/s de intervención.

4.2. Recursos disponibles: asociaciones, instituciones, especialistas...

4.3. Elaboración de criterios de selección y búsqueda de estrategias:

4.3.1. Acciones educativo sociales (comunes y compensatorias). Por ejemplo, búsqueda o construcción de métodos, instrumentos y técnicas concretas "ad casum".

4.3.2. Acciones dirigidas a la familia. Entre otros muchos criterios de estructuración, citamos:

- información (especificar, padres, otros...),

- formación (ej. ofrecer recursos de autoobservación),

- acciones terapéuticas (ej. entrenamiento de padres: revisión y modificación de relaciones aversivas, recursos no violentos, como tiempo fuera...

- acciones de apoyo económico (becas, etc.).

4.3.3. Acciones directas sobre los individuos en el ámbito personal, educativo (formativas, culturales, religiosas, profesionales...), social (urbanas, asistenciales,...), económicas o laborales. 
4.3.4. Acciones de apoyo comunitario (ej. de relación social con otros grupos humanos, asociaciones o instituciones de ocio, formativas, culturales, religiosas; interacción con otras instituciones,

Paso quinto: cambios conductuales que se pretenden, sean individuales, institucionales o relaciónales, y éstos en el individuo, en la familia, en la institución, en la comunidad, en el trabajo, en el ocio, etc..

- Ello requiere posibilitar todo aquello que permita comparar conductas antes y postintervención. Con el fin de poder evaluar o verificar tanto el proceso de ayuda al aprendizaje de la conducta objetivo, como el resultado del aprendizaje de conductas prosociales, laborales,etc.

Algunas actividades prácticas para conseguir el objetivo anterior son:

- Detectar y describir problemas conductuales.

- Evolución de la conducta:

- acciones de disminución de conductas inadecuadas, problemáticas, agresivas, antisociales...

- acciones para implementar conductas ya existentes

- corregir o eliminar conductas o comportamientos inadecuados

- implantar conductas deseables.

\section{Bibliografía}

ALBINUM, s. (1977). Teaching and personal relationships. Educational Theory, XXXVII, 4, 297-303.

BALL, S.J. (1989. La micropolítica de la escuela. Barcelona-Paidós.

BOUTIN, A. y DURNING, P. (1991). Intervenciones socioeducativas en el medio familiar. Madrid: Narcea

CASTAÑÉ, J. (1980). El fin social de la educación. Madrid: Bruño.

CASTAÑÉ, J. (1981). El concepto de educación como desarrollo intencional perfectivo. Revista Española de Pedagogía, 153 ,pág. 161.

COLOM,J. (1982). Teoría y metateoría de la educación. Mexico:Trillas.

FACULTAD DE FILOSOFÍA Y CIENCIAS DE LA EDUCACIÓN (1984). Programación docente de Ciencias de la educación. Madrid: Universidad Complutense. Facultad de Filosofía y Ciencia de la Educación, Programa de Pedagogía General del Departamento de Pedagogía Sistemática.

FERNÁNDEZ, P. (1991). La función tutorial. Madrid: Castalia.

FORO EUROPEO DE SEGURIDAD URBANA (1993). Urban cultural space and urban security. París: La Villete.

FRANTA, H. (1982). Interazione educativa. Roma:LAS.

GOLDSTEIN, A.P. (1991). El comportameinto agresivo. Madrid: Edit. Bibliotéca de Psicología.

IBAÑEZ MARTIN,J.A. (1989). Hacia una formación humanística. Barcelona:Herder.

JOVER, G. (1991). Relación educativa y relaciones humanas. Barcelona:Herder.

MARTIN RODRIGUEZ,J. (1993) Similarities in attitudes toward interpersonal aggression in Finland, Poland, and Spain, Journal o Social Psychology, 13: 737-739 (1993). 
MARTIN RODRIGUEZ, J. (1996) Aggression: causes and functions. Hiroshima Forum for Psychology, 17: 21-37 (1996)

MARTIN RAMIREZ J. \& FUIMARA,T. (1997). Cross-cultural study of attitudes toward interpersonal aggression. Ewansei Gakuin University Sociology Studies, 78: 97103 (1997).

MARTINEZ, A. (1995). El estudio de casos para profesionales de la acción social. Madrid:Narcea.

MEDINA, A. (1993) El clima social del centro educativo. Madrid:UNED.

MERINO, J.V. (1982). Problemas de base implicados en el proceso de integración social del deficiente mental. Bordón, 244, págs. 425-449.

MERINO, J.V. (1984). Socialización y educación. En Quintana Cabanas, J. Mạ. Sociología y economía de la educación. Madrid: Anaya.

MERINO,J.V. (1985). "El proceso de socialización en sujetos de Educación Especial: principios, líneas y programas de intervención educativa". Bordón, número 256, Enero-Febrero, 43-70.

MERINO, J. V. (1985). Funciones de la investigación-acción en las UU. PP. Revista Entre Líneas. $n^{\circ} 2$, págs. 75-89.

MERINO, J. V. (1987). Supuestos básicos para una pedagogía preventiva de la inadaptación y delincuencia juvenil. Bordón, nº 267, págs. 173-187.

MERINO, J. V. (1992). Acción preventiva de la inadaptación social. Revisión de planteamientos y perspectivas de futuro. En Muñoz Sedano, A. (Ed.) El educador social: profesión y formación universitaria. Madrid: Ed. Popular.

MORENO, M. (1997). Intervención psicoeducativa en las dificultades del desarrollo. Barcelona: Ariel Educación.

MUÑOZ SEDANO, A. (Ed.) (1992). El educador social: profesión y formación universitaria. Madrid:Popular.

ORTEGA ESTEBAN, J. (1992) La intervención comunitaria preventiva frente a la inadaptación social. Conferencia presentada en la Facultad de Educación de la Universidad Complutense durante la Semana de Educación Social.

QUINTANA, J. Ma. (1984). Pedagogía social. Madrid: Dykinson

QUINTANA, J. Ma. (1995) Teoría de la educación. Concepción antinómica de la educación. Madrid: Dykinson.

SABANZA, V. (1995). Progreso intelectual y social de los alumnos en la integración escolar. Tesis Doctoral. Madrid: UNED. Facultad de Educación.

STROMQUIST, N.P. (1996). "La violencia escolar en los Estados Unidos de América:tendencias, causas y respuestas", Perspectivas, 26 (1996), 391-416.

TRILLA, J. (1985). Notas para un modelo analítico de la acción educativa. Palma de Mallorca: Seminario Nacional sobre Teoría de la Educación.

WRIGHT, G.H. (1968). An easy in deontic logic and general Theory of action. Amsterdan.

WRIGHT, G.H. (1979). Norma y acción. Madrid: Tecnos. 